\title{
Improved shape for refinable surfaces with singularly parameterized irregularities
}

\author{
Kęstutis Karčiauskas ${ }^{a}$ and Jörg Peters ${ }^{b}$ \\ a Naugarduko 24, Vilnius University, LT-2006, Vilnius, Lithuania \\ ${ }^{b}$ Dept CISE, University of Florida, Gainesville FL 32611-6120, USA, jorg @ cise.ufl.edu, tel/fax 352.392.1200/1220
}

\begin{abstract}
To date, singularly-parameterized surface constructions suffer from poor highlight line distributions, ruling them out as a surface representation of choice for primary design surfaces. This paper explores graded, many-piece, everywhere $C^{1}$ singularlyparameterized surface caps that mimic the shape of a high-quality guide surface. The approach illustrates the trade-off between polynomial degree and surface quality. For bi-degree 5, minor flaws in the highlight line distribution are still visible when zooming in on the singularity, but the distribution is good at the macroscopic level. Constructions of degree bi-4 or bi-3 may require one or more steps of guided subdivision to reach the same macroscopic quality. Akin to subdivision surfaces, singularly-parameterized functions on the surfaces are straightforward to refine.
\end{abstract}
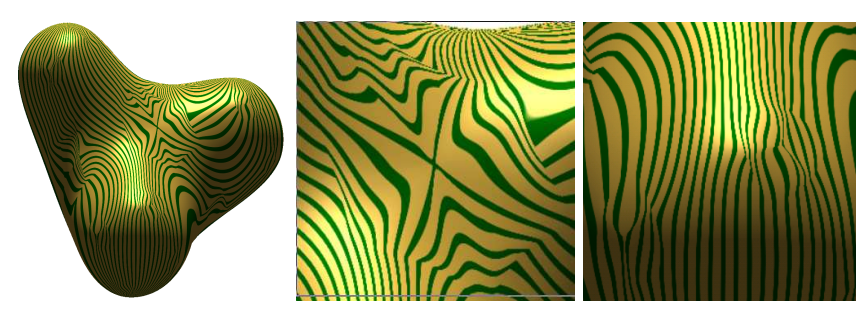

(a) old: Fig. 4b of [8]
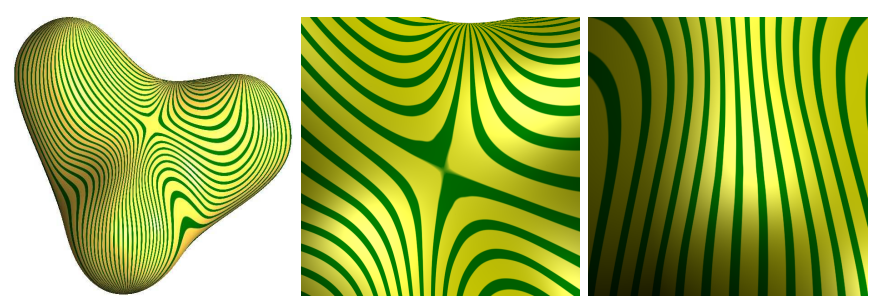

(b) new: this paper

Figure 1: Improved highlight line distribution: (middle) zoom on 6-valent and (right) on 3-valent irregularities.

\section{Introduction}

To directly support both design and analysis, a surface representation should offer both exact refinability and smooth multisided blends. Subdivision surfaces deliver both properties. But their representation as an infinite sequence of ever smaller smoothly-connected surface rings complicates their inclusion into existing industrial design infrastructure and the application of integration rules for analysis. Moreover, popular subdivision algorithms, such as Catmull-Clark-subdivision, often fail to deliver 'class A' surfaces. Fig. 12b illustrates a typical 'pinching' of highlight line distributions [1] in the neighborhood of an irregular node, already in the first steps at a macroscopic level. Geometrically smooth $\left(G^{k}\right)$ constructions fit directly into the $\mathrm{CAD}$ pipeline and have been shown to deliver class A shape; but refinement requires careful book-keeping to record what edges are $G$-connected in the initial surface. This paper explores a third option: singularly-parameterized freeform surfaces as presented in $[2,3,4,5,6,7,8]$. [8] demonstrated simple uniform refinability of singularly-parameterized surfaces for iso-geometric computations on the surfaces at different levels of resolution. However, its surfaces, as well as all the earlier ones, suffer from poor highlight line distributions near the singularities. Fig. 1a shows the shape to be typically worse than Catmull-Clark-subdivision.

Fig. 1b shows a much improved highlight line distribution for a new construction of degree bi-5. This is one of three options that yield singularly-parameterized surfaces of degree bi$s, s=3,4$ or 5 , that (i) are refinable, (ii) are by default everywhere at least $C^{1}$, and (iii) yield a good macroscopic highlight line distribution. The key to the improvement is mimicking a high-end guide surface $\mathrm{g}$ - by Hermite-sampling its composition with a special singular map. The resulting macroscopic highlight line distribution is satisfactory and more uniform than that of Catmull-Clark-subdivision. The microscopic flaws for constructions of degree bi-4 and bi-3 can be confined to an ever smaller region by guided subdivision based on $\mathrm{g}$. Guided subdivision does not incur the pinching and oscillations that CatmullClark-subdivision is prone to.

The constructions consist of two transition layers and a central cap. This layout anticipates refinement and has many free parameters. Although useful when selecting bivariate re-parameterizations, standard fairing-functionals derived from regular surfaces are unable to cope with the irregular distribution of many free parameters in 3-space and yield low-quality highlight line distributions for singularly-parameterized surfaces. By contrast, extensive experimentation shows that sampling the high-quality guide surface successfully reigns in excess degrees of freedom and prevents oscillations.

Overview. After a literature survey, Section 2 collects the notation and setup for constructing singularly-parameterized splines near irregularities. Section 3 defines the guide surface 
and sampling strategies and Section 4 presents constructions of bi-degree $s=5,4,3$. Section 5 compares their shape characteristics and Section 6 explains the simple, nested refinement of the corresponding analysis functions.

\subsection{Singular-parameterized surface constructions}

Since the 1990s, singular parameterization has been pointed out as a natural option to smoothly cover irregularities where more or fewer than four tensor-product (quadrilateral) surface pieces join. Singularly-parameterized $C^{1}$ surface constructions include [2, 3, 4, 5, 6, 7, 10]; [9] even shows how to construct $C^{2}$ surfaces (but specifies no rules that generically yield good shape). Independently, in the context of iso-geometry, Takacs and Jüttler [11] discussed singular spline constructions for analysis and observed that specific linear combinations of singular splines can be sufficiently regular for iso-geometric analysis. The monograph [12] characterizes subdivision surfaces as smooth spline surfaces with singularities at the irregular points and establishes the differential-geometric properties of subdivision surfaces at the singularities. Subdivision functions have repeatedly been used as finite elements $[13,14,15,16]$. A major contribution of [7] to the theory of singularly-parameterized surfaces is a proof that the corner singularity of this bi-3 construction is removable by a localized change of variables. This proves tangent continuity at and near the irregularity. [8] presents a singularly-parameterized construction with four free control points per quad. However, the highlight line distribution of this simple uniformly-refinable $C^{1}$ construction are often pinched or otherwise irregular near the singularity, indicating insufficient quality for use as outer surfaces.

\section{Definitions and Setup}

Since the aim is to improve the shape of constructions such as [8], we consider the same type of input mesh whose nodes are interpreted as B-spline-like control points. That is, we consider as input a network of quadrilateral facets or quads. The nodes where four quads meet are called regular, else irregular nodes. The 2-ring of quads surrounding an irregular node forms a cnet (see Fig. 2) with $n \neq 4$ sectors. Good quad-mesh design and automatic quad-mesh generation avoid adjacent irregular nodes. If an irregular node nevertheless shares a quad with another irregular node, one local Catmull-Clark-refinement step separates c-nets (see e.g. Fig. 17a). The resulting T-joints are, as always for Catmull-Clark subdivision, compatible with the splines of the coarser quad-mesh. The c-net of Fig. 2a defines the characteristic map $\chi$ of Catmull-Clark [12] for $n=5$.

Surface and analysis space are formed as linear combinations $\sum \mathbf{c}_{\alpha} f_{\alpha}$ with control points $\mathbf{c}_{\alpha}$ and basis functions $f_{\alpha}$ obtained by setting $\mathbf{c}_{\alpha}=1$ and all other coefficients to zero and then applying the construction of Section 4 . We will use piecewise tensor-product polynomials $\mathbf{b}$ of bi-degree $s$ in BernsteinBézier (BB) form

$$
\mathbf{b}(u, v):=\sum_{i=0}^{s} \sum_{j=0}^{s} \mathbf{b}_{i j} B_{i}^{s}(u) B_{j}^{s}(v), \quad(u, v) \in \square:=[0 . .1]^{2},
$$

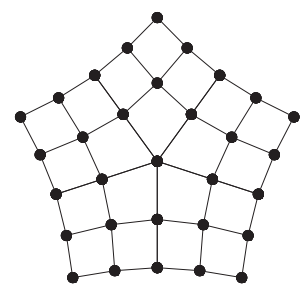

(a) c-net

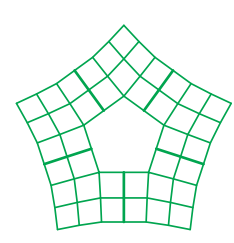

(b) boundary data
Figure 2: Input quad mesh. (a) A net of B-spline-like control points $\mathbf{c}_{\alpha}$ defines cross-boundary derivatives up to and including order 2 ; the derivatives are expressed in (b) in BB-form of degree 3 with coefficients $\mathbf{b}_{i j}^{k}$.

where $B_{k}^{s}(t):=\left(\begin{array}{l}s \\ k\end{array}\right)(1-t)^{s-k} t^{k}$ are the Bernstein-Bézier (BB) polynomials of degree $s$ and $\mathbf{b}_{i j}$ are the BB coefficients [17, 18]. We use the corner jet constructor

$$
[\phi]_{j \times j}^{s}\left(u_{0}, v_{0}\right)
$$

that expresses the expansion of a function $\phi$ at $\left(u_{0}, v_{0}\right)$ up to order $j$ in $u$ and in $v$ in BB-form of degree bi-s, i.e. by $j \times j$ BB-coefficients.

\section{Guide Surface Construction}

To facilitate parametric continuity and refinement of the singular construction, we re-approximate surfaces $\mathbf{G}$ of [19] by guide surfaces $\mathbf{g}$. Both $\mathbf{G}$ and $\mathbf{g}$ are of degree bi-5 in each of $n$ sectors, are $G^{1}$-connected and have a well-defined quadratic expansion $\mathbf{q}$ at the irregular point in the center. The derivation and construction of $\mathbf{G}$ is non-trivial - but no deep understanding of [19] is required to follow the present construction.

We can also derive and represent $\mathbf{g}$ as a linear combination of pre-computed, tabulated generating functions. The generating functions are B-spline-like, i.e. smooth and of local support, and when weighted with the nodes $\mathbf{c}_{\alpha}$ of a $\mathbf{c}$-net, yield the surface.

Let $\sigma: \mathbb{R}^{2 \times n} \rightarrow \mathbb{R}^{2}$ be the $2 \pi / n$-rotationally symmetric map obtained by applying the algorithm of [19] to the control points of $\chi$ (cf. Fig. 2a). Fig. 3c, left, shows one sector of $\sigma$ for $n=5$. Denote by $L$ the linear shear (Fig. 3b) that maps the unit square to the unit parallelogram with opening angle $\frac{2 \pi}{n}$. Finally, denote by $\mathbf{f}: \mathbb{R}^{2 \times n} \rightarrow \mathbb{R}$ a map of total degree 5 that is $C^{1}$, has six coefficients (black disks in Fig. 3a) determining a unique quadratic expansion at the center (grey region), and six coefficient (red disks) per sector that can be chosen freely and that uniquely pin down the coefficients along the sector separator lines when enforcing $C^{1}$ continuity between sectors.

We set $\mathbf{g}:=\mathbf{f} \circ L$ except that we retain as free the $4 \times 4$ coefficients (shown as black disks Fig. 3b, right) whose BB-functions vanish up to first order along the sector separating lines, hence do not influence smoothness between sectors. Setting the central point of $\mathbf{g}$ to the central point of $\mathbf{G}$, we collect the just-listed $(6-1)+6 n+(4 \times 4) n$ unconstrained coefficients of $\mathbf{g}$ in to a set $\mathcal{G}$. By sampling each sector $\mathbf{g}_{i}$ according to $\left[\mathbf{g}_{i} \circ L^{-1} \circ \sigma\right]_{3 \times 3}^{5}$ at the four corners of the unit square domains of $\sigma$, we obtain BB-coefficients $\mathbf{b}_{i j}^{k}, k=0, \ldots, n-1, i, j=0, \ldots, 5$. The $\mathbf{b}_{i j}^{k}$ 


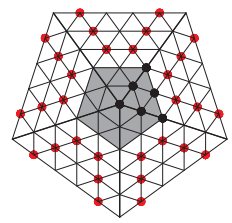

(a) $C^{1}$ map $\mathbf{f}$ of total degree 5
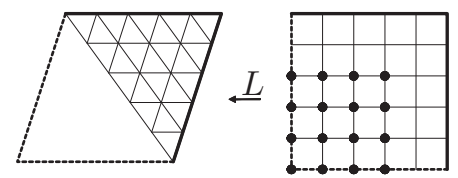

(b) map $L$
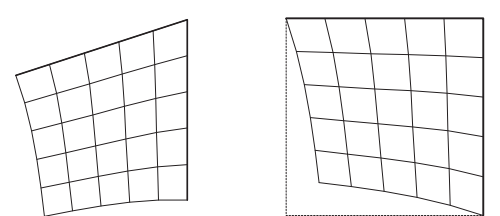

(c) sector of $\sigma$ and its image under $L^{-1}$

Figure 3: Guide surface construction. (a) Control net of the auxiliary map $\mathbf{f}$ of total degree 5. Coefficients involved in $C^{1}$ continuity across sector lines are marked as red disks if they are free after enforcing $C^{1}$ continuity. (c,left) BBcoefficients of one sector of $\sigma$ obtained by applying the construction of [19] to Fig. 2a; right: its linear transformation by the shearing map $L$ in (b). In (b,c) the origin is the upper right hand corner.

are linear in the coefficients of $\mathcal{G}$ and can be assembled to form $n$ patches $\mathbf{b}^{k}$ of degree bi-5. Then $\mathcal{G}$ is determined as the leastsquares solution, minimizing the sum of differences between each $\mathbf{b}_{i j}^{k}$ and its corresponding coefficient of $\mathbf{G}$.

\subsection{Sampling map $\chi$ for guided subdivision}

Sampling $\mathrm{g}$ with respect to $L^{-1} \circ \chi$ (see Fig. 4) allows decreasing the region of the singular construction by inserting nested surface rings before applying one of the constructions in the next section. Since copies of $\chi$ scaled by the sub-dominant eigenvalue $\lambda$ of CatmullClark-subdivision join $C^{2}$ and $\chi$ has rotational symmetry it suffices to store the sampling for the generating functions of $\mathbf{g}$ at the three circled locations in Fig. 4. $C^{2}$-connected bi-5 surface rings can then be built as laid out in Section 4.1 or $C^{1}$-joined bi-3 or bi- 4 rings as explained in Section 4.2. For a deeper discussion of guided subdivision see [20].

\subsection{Singular sampling map $\tau$}

To sample the guide for the singular construction, we use a reparameterization $\tau$. The map $\tau$ consists of $n$ copies, rotated by $2 \pi / n$, of a $4 \times 4$ bi-3 patchwork illustrated in Fig. 5a for $n=5$. The patchwork is symmetric with respect to its bisectrix. The outermost layers of BB-coefficients (green in Fig. 5a) match the derivatives of the characteristic map $\chi$ and the BBcoefficients on sector-separating lines are perpendicular projections of the adjacent layer so that rotated copies join $C^{1}$. Gray underlay indicates coefficients pinned down to enforce internal $C^{1}$ transitions. The six layers between the green and the gray layers are selected to form a $C^{2}$ piecewise cubic map interpolating second-order data (one stencil, for the end point indicated by a rectangle, is displayed in Fig. 5a top-left; to be divided by 6 .) The BB-coefficients of $M$ are established by the same operation once its neighbor bi-3 pieces are determined.

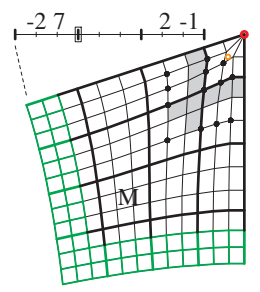

(a) singular 16-piece parameterization $\tau$ (right: zoom of singularity)

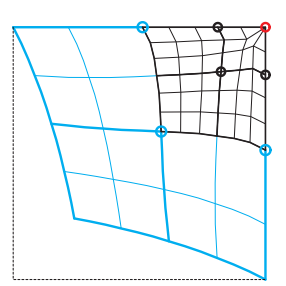

(b) $L^{-1} \tau$
Figure 5: Singular sampling parameterization. (a) The red circle marks the origin, green position, first and second derivatives (in bi-3 BB-form) of the characteristic map $\chi$ of Catmull-Clark subdivision. (b) Seven locations (marked as cyan, black and red circles) will be used for sampling Hermite data.

Due to symmetry, the bivariate map has 14 scalar unknowns that define the unconstrained BB-coefficients marked as black disks and the ochre circle $\circ$ of the singular bi-3 piece. The central point coincides with its three neighbors. The coefficient marked $\times$ is the perpendicular projection of $\circ$ and (cf. Fig. 5a, right)

$$
\bullet:=t_{n} \circ+\left(1-t_{n}\right) \times, \text { where } t_{n}:=\frac{3}{20}+\frac{2}{3} \cos \frac{\pi}{n} .
$$

(This choice simplifies refinement.) The 14 unknowns are determined by minimizing the (sum of) functional(s) $\mathcal{F}_{2}$ applied to all 16 patches and both coordinates, where $\mathcal{F}_{k} f:=\int_{0}^{1} \int_{0}^{1} \sum_{i+j=k, i, j \geq 0} \frac{k !}{i ! j !}\left(\partial_{s}^{i} \partial_{t}^{j} f(s, t)\right)^{2} d s d t$. Comparison for $k=1,2,3,4$ showed $\mathcal{F}_{2}$ to yield the most uniform control net (see Fig. 6 for $n=5$ ).
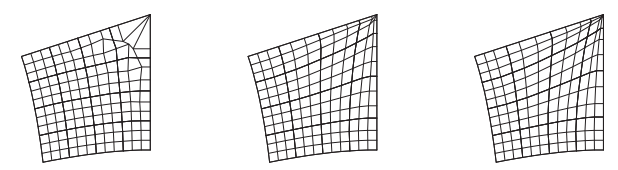

Figure 6: Not selected alternatives to the map $\tau$ (Fig. 5a): left minimizing $\mathcal{F}_{k}$ for $k=1$; middle $k=3$; and right $k=4$.

\section{Construction of singularly parameterized surfaces}

Fig. 7 shows the basic structure of the singular construction: there are always two Catmull-Clark-like transition rings that consist of six patches $(\mathrm{A}, \mathrm{B}, \mathrm{C}, \mathrm{D}, \mathrm{E}, \mathrm{F})$ plus a central patch that is $2 \times 2$ split for degree bi- $s, s \in\{3,4,5\}$. Since there is a tradeoff between the degree and the quality of the resulting surface, we lay out three options of bi-degree 5,4 and 3 plus an auxiliary bi- 6 construction that simplifies exposition and provides data to the split patches. 


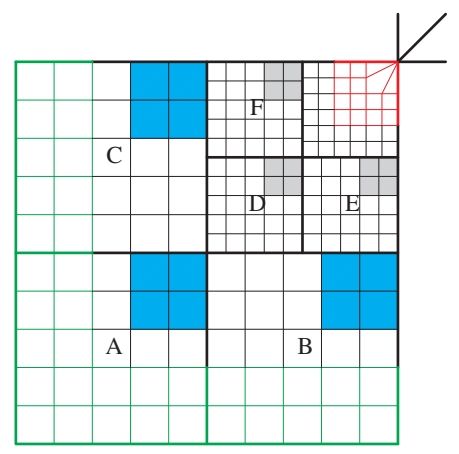

Figure 7: Auxiliary singular bi-6 patch and bi-5 transition layer.

\subsection{Bi-5 construction and auxiliary bi-6 construction}

We first consider the transition layers. An outer transition ring of degree bi-5 is determined by $C^{2}$-extending the outside patches (outer green layers) and by sampling $\left[\mathrm{g} \circ L^{-1} \tau\right]_{3 \times 3}^{5}$ at the three cyan locations shown in Fig. 5b. The latter determines three $3 \times 3$ BB-coefficients of A,B,C (cyan-underlaid in Fig. 7a). $C^{2}$ prolongation joins $\mathrm{A}$ to $\mathrm{B}$ and $\mathrm{C}$. By construction of $\mathrm{g} \circ$ $L^{-1} \tau$, the sectors join $C^{1}$; to join $C^{2}$ all coefficients next to the boundary are adjusted (hence preserving $C^{2}$ continuity in the direction along the boundary). The second transition layer is determined analogously by sampling at three black locations in Fig. 7a (grey-underlay), $C^{2}$ adjustment between sectors and $C^{2}$ extension inward of A,B,C according to the refined layout.

We complete the auxiliary bi-6 construction using one bi-6 patch for each of the $n$ sectors (see Fig. 7a). To reproduce, at the central point, the quadratic expansion of $\mathrm{g}$, the singular bi-6 patches sample $\left[\mathrm{g} \circ L^{-1} \tau\right]_{5 \times 5}^{6}(0,0)$ (red mesh in Fig. 7a). The bi-6 patches are then fully determined by $C^{1}$-prolongation of patches D,E and F.

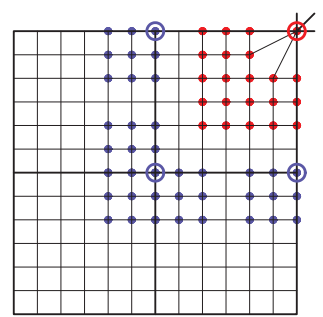

(a) $2 \times 2$ split bi- 6 cap $\mathbf{p}$

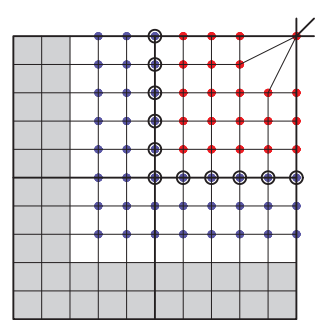

(b) construction of bi-5 macro-patch
Figure 8: Bi-5 cap construction: innermost $2 \times 2$ macro-patch. (a) split of auxiliary bi- 6 patch and collection of Hermite data; (b) transformation of the Hermite data to bi-5 form $[\mathbf{p}]_{3 \times 3}^{5}$ at three locations; transformation to $[\mathbf{p}]_{5 \times 5}^{5}(0,0)$ at the center; and internal smooth join.

For the bi-5 construction, see Fig. 8, the bi-6 patch $\mathbf{p}$ is split $2 \times 2$ and $[\mathrm{p}]_{3 \times 3}^{5}$ of the singular piece is sampled with the correct level of refinement at the three outer corners, marked as blue circles. $C^{2}$-prolongation of the patches D,E,F (gray region in Fig. 8) defines the remainder of the patches. For the center piece, $[\mathbf{p}]_{5 \times 5}^{5}(0,0)$ determines the red BB-coefficients. The circled BB-coefficients are re-defined as averages of blue and red disks to ensure internal $C^{1}$ connectivity. By construction, adjacent sectors are already $C^{1}$ connected.

\subsection{Bi-3 and bi-4 constructions}

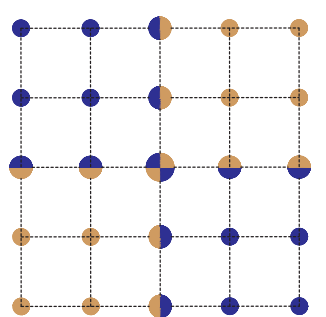

(a) averaging $3 \times 3$ data

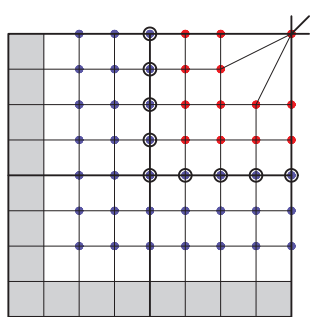

(b) central $2 \times 2$ bi- 4 cap
Figure 9: Bi-4 cap construction.

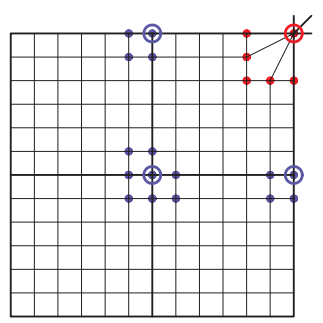

(a) sparse Hermite sample

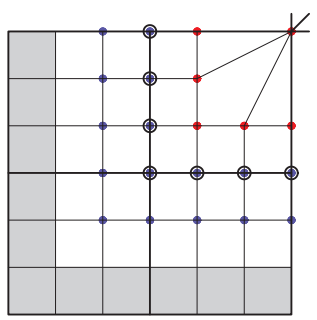

(b) central $2 \times 2$ bi- 3 cap
Figure 10: Bi-3 construction: (a) collection of Hermite data from the bi- 6 auxiliary patch $\mathbf{p}$ for (b) transformation to the central $2 \times 2$ bi- 3 cap.

The bi-3 and bi-4 constructions are structurally alike. Hermite data are sampled in bi-s form, $s=3,4$, as $[\mathbf{p}]_{s-1 \times s-1}^{s}$ at the same corners of the transition patches $A, B, C, D, E$, $F$ as in the earlier bi-5 construction. For $s=4$ this requires averaging $[\mathbf{p}]_{3 \times 3}^{4}$ data at overlapping locations as illustrated in Fig. 9a. The two transition layers are $C^{1}$-connected by prolongation. Sampling $[\mathbf{p}]_{3 \times 3}^{4}$ at the three outer corners of the singular patch, and $[\mathbf{p}]_{4 \times 4}^{4}$ at the center, $C^{1}$ prolongation from D,E,F (gray regions) and enforcing internal $C^{1}$ continuity by setting the coefficients displayed as circles as averages of their blue and red neighbors completes the construction. Since the central sample consists of only $4 \times 4$ coefficients (red in Fig. 9b), bi-4 surfaces do not preserve a quadratic at the center. The bi-3 surface construction collects still less data from p (see Fig. 10a) resulting in a further deterioration of the highlight line distribution as the next section will show.

The constructions require no injectivity proof as in [7] since the guide surface $\mathrm{g}$ is non-singular and the sampling of the composition with the singular reparameterization preserves the linear (for bi-5: quadratic) expansion of $\mathbf{g}$ at the center point.

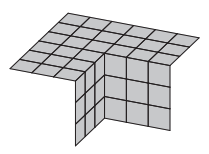

(a) $n=5$

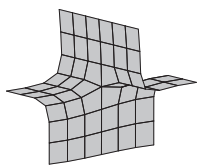

(b) $n=6$

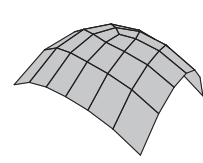

(c) $n=3$

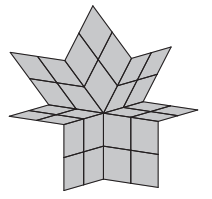

(d) $n=7$
Figure 11: Some extend c-nets used for comparisons. 


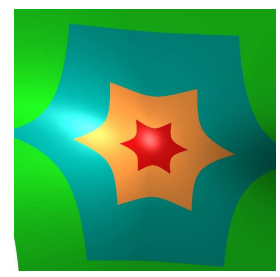

(a) no subdivision

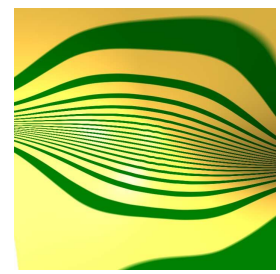

(c) bi-5

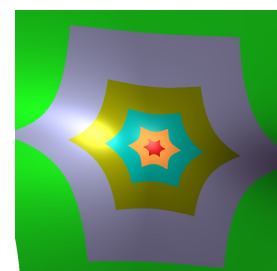

(f) $2 \times$ subdivision

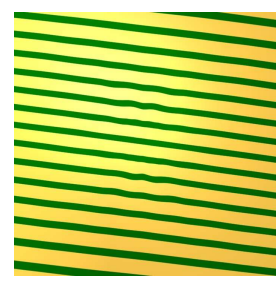

(i) bi-5

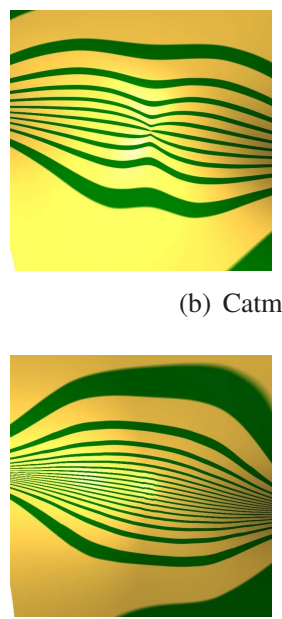

(d) bi-4

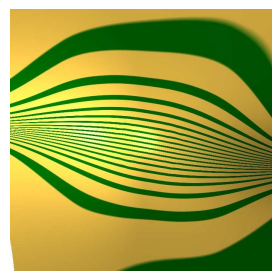

(g) bi-4

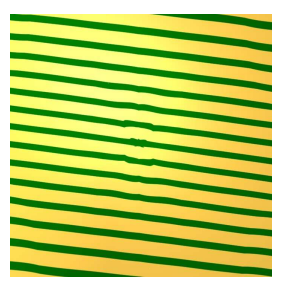

(j) bi-4
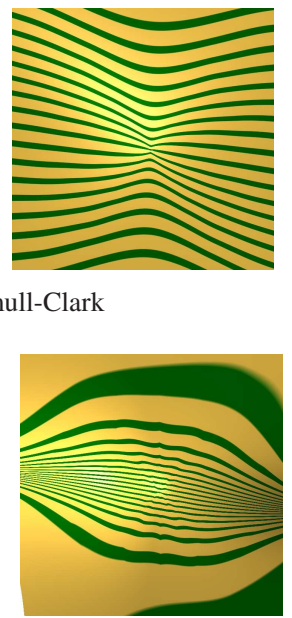

(e) bi-3

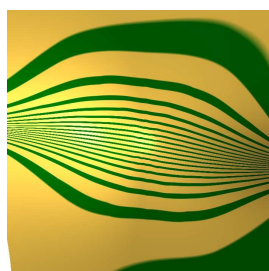

(h) bi-3

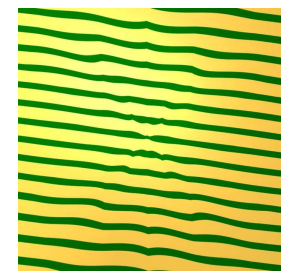

(k) bi-3
Figure 12: Surfaces for c-net Fig. 11b. 3rd and 4th row: after two steps of guided subdivision. (i), (j), (k) zoom to the innermost two layers (orange, red)

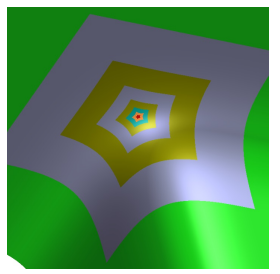

(a) $4 \times$ subdivision

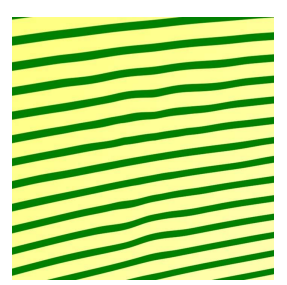

(d) bi-5

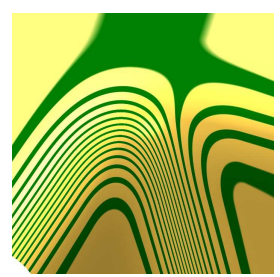

(b) bi-4

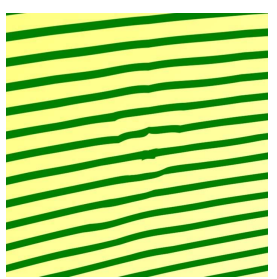

(e) bi-4

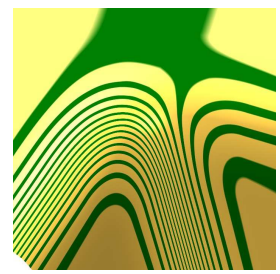

(c) bi-3

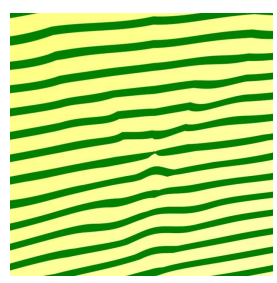

(f) bi-3
Figure 13: Surfaces from c-net Fig. 11a + four steps of guided subdivision. (d),(e),(f) zoom to innermost two layers.

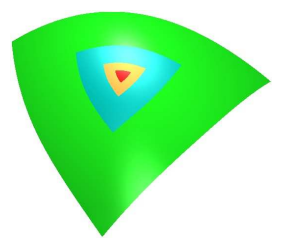

(a) $n=3$

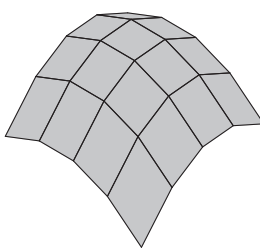

(d) $n=4$ net

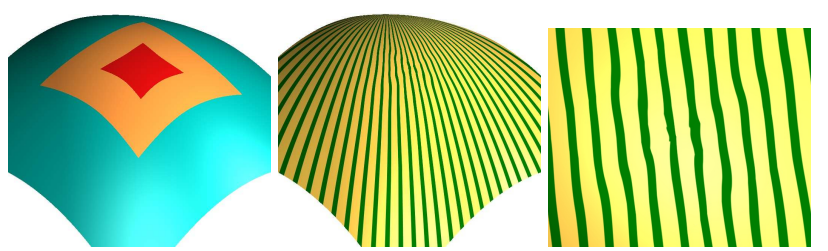

(f) $n=4$ bi- 3 construction

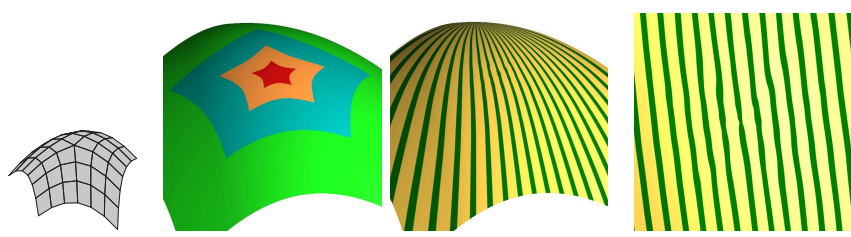

(g) $n=5$

(h) $2 \times$ subdivision

(i) zoom

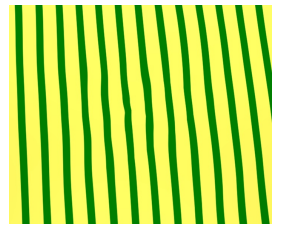

(c) zoom

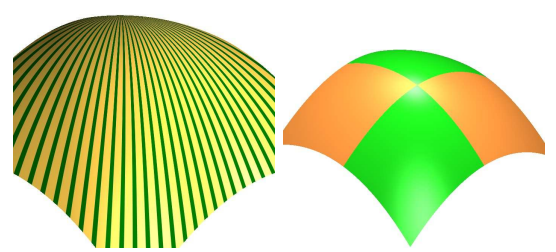

(e) bi-3 spline guide
Figure 14: (a,b) $n=3$ bi-3 surface (no guided subdivision step) from c-net Fig. 11c. (d,e) regular bi-3 spline surface and (f) singularly-parameterized approximation; last row: $n=5$. All zoom is to the inner two layers (orange, red).

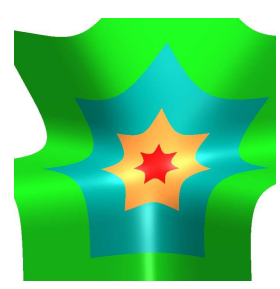

(a) Fig. 11d: $n=7$

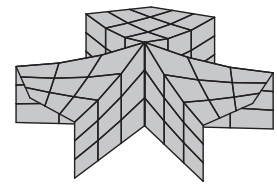

(d) $n=9$

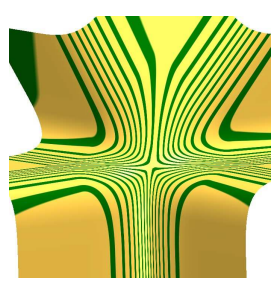

(b) bi-5

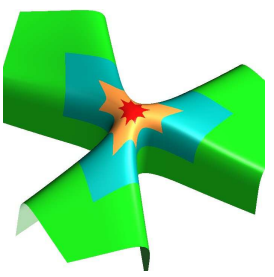

(e)

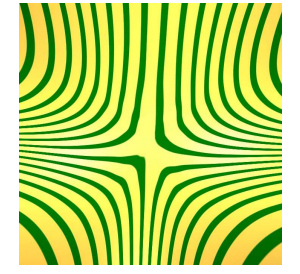

(c) zoom

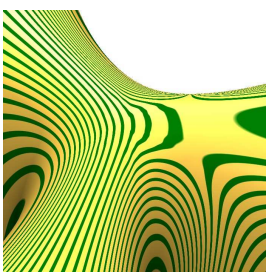

(f)
Figure 15: Higher valences. All zoom is to the inner two layers (orange, red). 


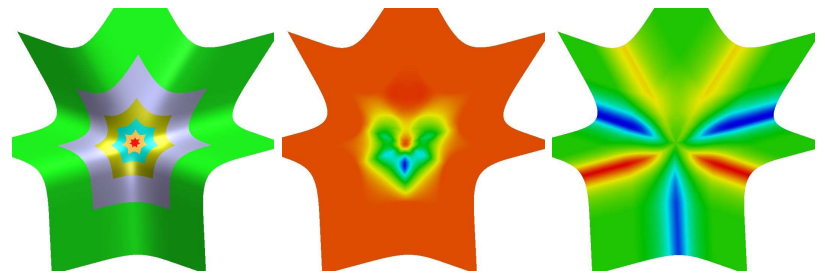

Figure 16: Gauss and mean curvature of the bi-5 construction for input Fig. 11d after two steps of guided subdivision.

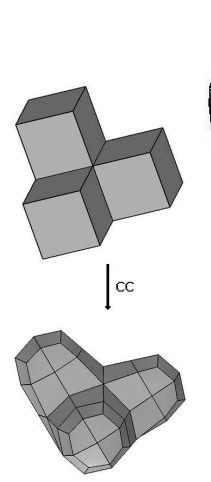

(a)
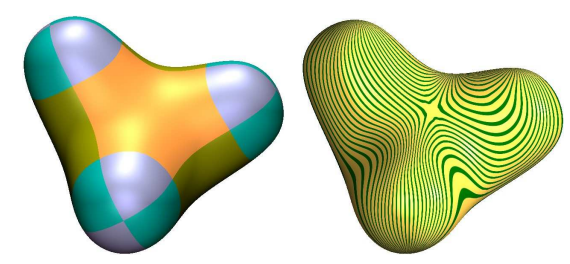

(c) bi-3
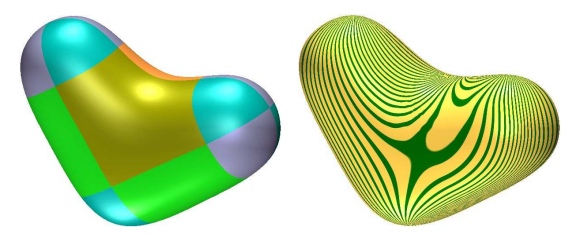

(e) bi-3
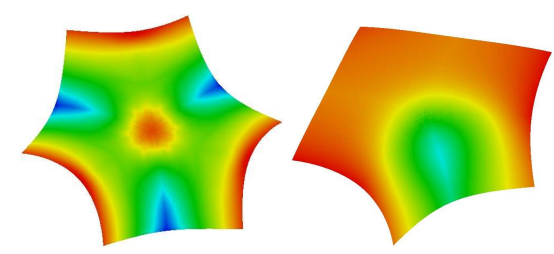

(g) Gauss curvature, bi-5

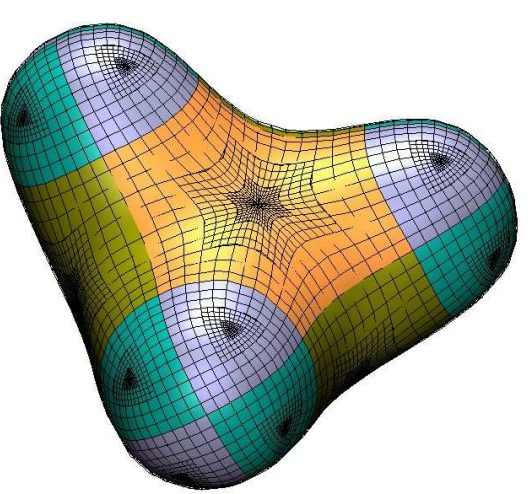

(b) bi-3 BB-net

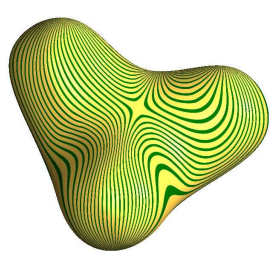

(d) bi-4

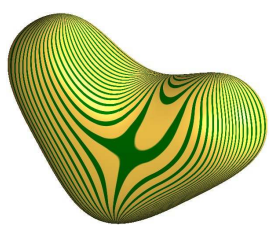

(f) bi-4

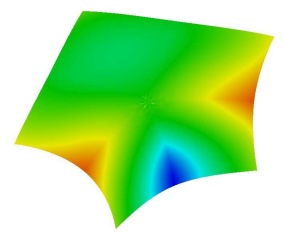

(h) mean curvature, bi-5
Figure 17: Surface layout and highlight line distribution of constructions applied to the once-Catmull-Clark-refined mesh (a). (g), (h) illustrate bi-5 constructions of the most challenging neighborhoods.

\section{Comparison of the constructions}

We focus on highlight lines for comparing the bi-5, bi-4 and bi-3 surfaces (bi-6 and bi-5 highlight lines look alike and only curvature shading differs slightly.) For exposed surfaces, experts and consumers associate oscillating or pinched highlight lines not only with a poor surface quality, but also with poor quality of the product since, beyond the bad visual impression, surface oscillations can impact the flow around the object and may indicate worse touch or elastic properties.

Fig. 11 shows a subset our test c-nets. Each is augmented by one ring of quads to define one bi-cubic surrounding surface ring (green in the examples). None of the tested examples (and many other) revealed artifacts between the surrounding surface and within the two transition rings, A,B,C (blue) and D,E,F (orange) in Fig. 7. The central $2 \times 2$ cap is red. In the following, enlargements zoom into the two innermost rings, orange and red.

Fig. 12 illustrates the decay of quality with lower degree. Bi4 and bi-3 surfaces do not have a unique quadratic at the center and preserve only a linear expansion of the guide g. Fig. $12 \mathrm{~g}, \mathrm{~h}$ demonstrate how applying two steps of guided subdivision before the constructions improves the macroscopic highlight line distribution, even for a $C^{1}$ construction of degree bi-3. Magnification of the central area, however, reveals that the characteristic shape flaws have just been compressed to a smaller area. Fig. 13d,e,f reveal similar oscillations after four steps of guided subdivision for surfaces of valence 5 . These oscillations are not visible at the macroscopic scale Fig. 13b,c, or even at the zoom level of Fig. 12i,j,k, and we do not expect this to be a problem in practice since real world applications come with a threshold on manufacturing fidelity: sheet metal will not replicate features at the micro-meter range. Repeated shape-preserving subdivision therefore eliminates the flaws for practical purposes. Bi-5 surfaces have a well-defined curvature at the singularity so that tracing on the surface is not a problem.

Fig. 14a,b,c are representative for our finding that singular parameterization works well for valence 3 . There is of course no need to test the case $n=4$, but Fig. 14d,e,f take advantage of the regular case to illustrate the effect of singular parameterization by using the regular bi-3 spline patches as guide. Fig. 14g adds a third positive test for the convex shape, where even small defects are often visible.

Fig. 15 illustrates that high valences up to $n=10$ pose no challenge to the constructions. Fig. 16 displays the curvature of the saddle defined by Fig. 11d. Fig. 17 combines irregularities of valence $n=3,5,6$.

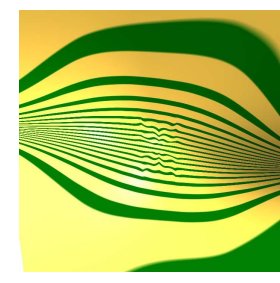

(a) no subdivision

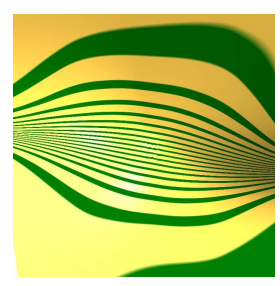

(b) $2 \times$ subdivision
Figure 18: Rejected bi-5 analogue of bi-6 construction.

\subsection{Default choices and rejected alternatives}

Preserving the unique (singularly reparameterized) quadratic expansion of the guide at the central point yields a uniform 

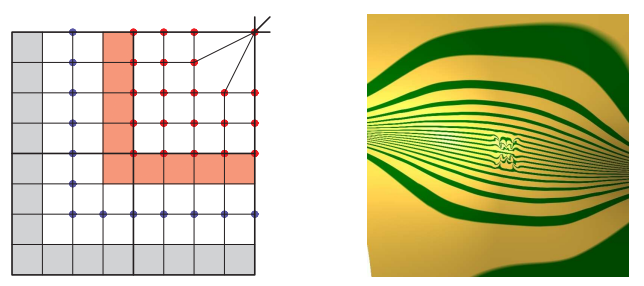

Figure 19: Rejected bi-4 construction with well-defined curvature at the center by sampling $[\mathbf{p}]_{5 \times 5}^{4}$ and $C^{1}$ extension outwards ('light red' underlay).

highlight line distribution. Unlike the bi-6 construction though, the default construction splits the central bi-5 cap $2 \times 2$. A single bi-5 patch can pick up the curvature at the central point and connect $C^{1}$ to the inner transition layer $\mathrm{D}, \mathrm{E}, \mathrm{F}$, but results in the unsatisfactory highlight line distribution of Fig. 18a. Fig. 18b shows that guided subdivision fails to improve the central highlight line pattern (compare to Fig. 13d). A bi-5 construction of the same quality as the default one can be obtained by $2 \times 2$ splitting of $L^{-1} \tau$ (Fig. 5b) before composing with $\mathrm{g}$ and sampling. However, the default construction facilitates implementation via smaller pre-computed tables and allows us to present the simpler bi- 6 construction that may be admissible in some applications. Sacrificing quadratic reproduction at the center, the transition rings can $C^{2}$-connect to an un-split singular central bi-5 cap. This variant yields a reasonable highlight line distribution.

Fig. 19 illustrates an alternative that preserves the full quadratic at the center also for a bi-4 construction. This implies that the central patches are singularly reparameterized quadratics. The resulting stiffness is known to negatively affect highlight lines and this flaw is emphasized under singular parameterization to the detriment of shape, see Fig. 19b.

Exploring one rather than two transition rings resulted in poor highlight line distributions even for degree bi-5 (see Fig. 20), even when combined with guided subdivision.

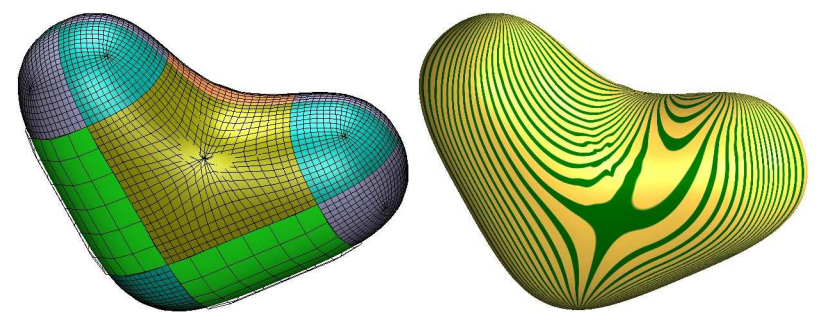

Figure 20: Rejected bi-5 construction using a single transition layer.

\section{Refinement of functions on surfaces}

The main goal of this paper is to improve the shape of singularly parameterized surfaces so one can design with them and additionally take advantage of the $C^{1}$ refinability of structurally identical functions on the surfaces. Steps of guided subdivision are only used to shrink the irregular neighborhood while closely following the guide surface - not for exact refinement. Refinement of the space of functions on the surface, e.g. for isogeo- metric design, is mostly straightforward: regular patches, e.g. transition rings and non-singular patches of the central cap, admit regular B-spline subdivision of $C^{1}$ splines, (except for the $C^{1}$ correction in the bi-3 construction, Fig. $10 \mathrm{~b}$.

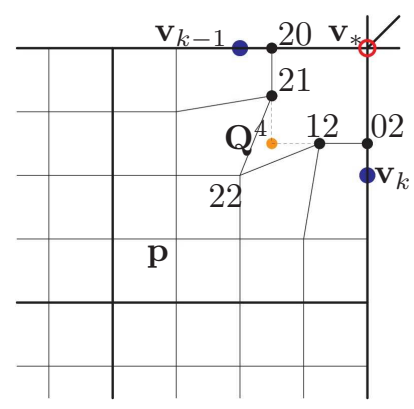

(a) before refinement

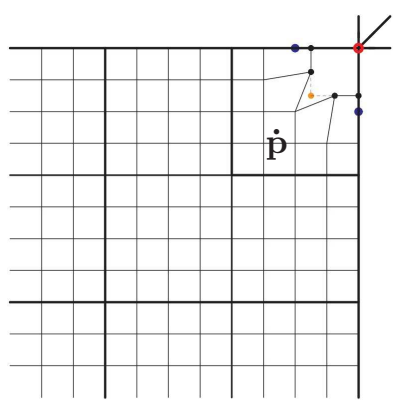

(b) after refinement
Figure 21: Refinement. The points $\mathbf{v}_{*}$ and $\mathbf{v}_{k}, k=0, \ldots, n-1$ are related by (2) and form the tangent plane. In (b) only $\dot{\mathbf{p}}$ is marked; $\dot{\mathbf{Q}}^{4}$ is the ochre point and the $\dot{\mathbf{v}}_{k}$ are blue bullets.

Only the central singular patch $\mathrm{p}$ requires a closer look. Fig. 21 illustrates the bi-4 case. The focus is on the BBcoefficients $\mathbf{p}_{02}, \mathbf{p}_{12}, \mathbf{p}_{21}, \mathbf{p}_{20}$ (indexed so that $\mathbf{p}_{00}=\mathbf{p}_{01}=$ $\mathbf{p}_{10}=\mathbf{p}_{11}=\mathbf{v}_{*}$ ) that influence tangent plane continuity at the central point.

With $t_{n}$ defined by (1), we initialize

$$
\begin{aligned}
& t^{s}:=\left\{\begin{array}{ll}
\frac{3}{10} t_{n}, & s=5, \\
\frac{3}{8} t_{n}, & s=4, \\
\frac{1}{2} t_{n}, & s=3,
\end{array} \quad \eta^{s}:=\left\{\begin{array}{ll}
\frac{3}{10}, & s=5, \\
\frac{1}{2}, & s=4, \\
1, & s=3,
\end{array} \quad e^{s}:=\eta^{s} \mathrm{c},\right.\right. \\
& \mathbf{v}_{k}:=\left(1-\frac{1}{e^{s}}\right) \mathbf{v}_{*}+\frac{1}{e^{s}} \mathbf{p}_{02}, k=0, \ldots, n-1, \quad \mathrm{c}:=\cos \frac{\pi}{n} \text {. }
\end{aligned}
$$

Since $\mathbf{p}_{02}, \mathbf{p}_{12}, \mathbf{p}_{21}, \mathbf{p}_{20}$ are the result of a singularly reparametrized linear expansion at $\mathbf{v}_{*}$,

$$
\mathbf{v}_{k+1}=-\mathbf{v}_{k-1}+2 \mathbf{c} \mathbf{v}_{k}+2(1-\mathbf{c}) \mathbf{v}_{*}
$$

holds.

The refinement leaves the central point $\mathbf{v}_{*}$ unchanged. The refinement relations

$$
\begin{aligned}
\dot{\mathbf{v}}_{k} & :=\frac{3}{4} \mathbf{v}_{*}+\frac{1}{4} \mathbf{v}_{k}, \\
\dot{\mathbf{Q}}^{s} & :=\left(1-2 d^{s}\right) \mathbf{v}_{*}+d^{s}\left(\dot{\mathbf{v}}_{k-1}+\dot{\mathbf{v}}_{k}\right), \quad d^{s}:=\frac{\eta^{s}}{2 c} \\
\dot{\mathbf{p}}_{02} & :=\left(1-e^{s}\right) \mathbf{v}_{*}+e^{s} \dot{\mathbf{v}}_{k}, \\
\dot{\mathbf{p}}_{12} & :=\dot{t}^{s} \dot{\mathbf{Q}}^{s}+\left(1-\dot{t}^{s}\right) \dot{\mathbf{p}}_{02}, \quad \dot{t}^{s}:=t^{s} / 2,
\end{aligned}
$$

( $\dot{\mathbf{p}}_{20}$ and $\dot{\mathbf{p}}_{21}$ are defined by symmetry) stem from a $2 \times 2$ split (de Casteljau's algorithm applied at $u=1 / 2=v$ ) of the singularly parameterized patches, hence guarantee exact reproduction of the original surface. Besides the new degrees of freedom from the new ring of regular patches, free are also $n+3$ points $\dot{\mathbf{v}}_{*}, \dot{\mathbf{v}}_{0}, \dot{\mathbf{v}}_{1}$ and $\dot{\mathbf{p}}_{22}$ in each sector.

Fig. 22 illustrates refined functions by embossing the singularly-parameterized surfaces taking advantage of the layout. 


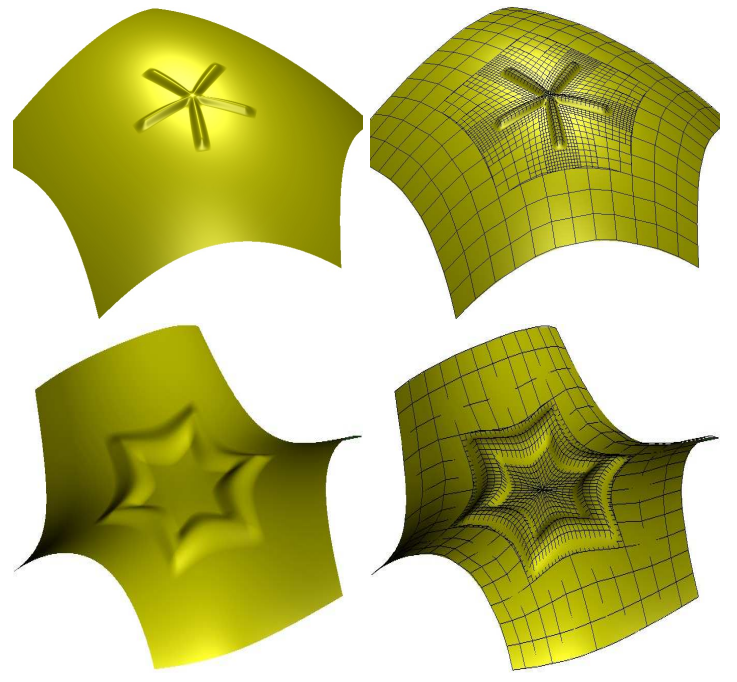

Figure 22: Refinement for local displacement.

\section{Conclusion}

Three alternative $C^{1}$ multi-sided cap constructions, of degree bi- $s$ for $s=5,4,3$, were presented. Each has characteristic microscopic flaws in the highlight line distribution. These are not easily visible at the macroscopic level. In fact, the bi-5 construction has a well-defined curvature at the center point. The resulting surfaces admit compatible $C^{1}$ functions for analysis or displacement that are easily refinable.

Acknowledgements. This work was supported in part by NSF grant CCF-1117695 and NIH R01 LM011300-01.

[1] K.-P. Beier, Y. Chen, Highlight-line algorithm for realtime surface-quality assessment, Computer-Aided Design 26 (4) (1994) 268-277.

[2] J. Peters, Parametrizing singularly to enclose vertices by a smooth parametric surface, in: S. MacKay, E. M. Kidd (Eds.), Graphics Interface '91, Calgary, Alberta, 3-7 June 1991: proceedings, Canadian Information Processing Society, 1991, pp. 1-7.

[3] J. D. Warren, Multi-sided rational surface patches with independent boundary control, in: IMA Conference on the Mathematics of Surfaces, 1992, pp. 281-294.

[4] P. R. Pfluger, M. Neamtu, On degenerate surface patches, Numerical Algorithms 5 (11) (1993) 569-575.

[5] M. Neamtu, P. R. Pfluger, Degenerate polynomial patches of degree 4 and 5 used for geometrically smooth interpolation in 3, Computer Aided Geometric Design 11 (4) (1994) 451-474.

[6] U. Reif, A note on degenerate triangular Bézier patches, Computer Aided Geometric Design 12 (5) (1995) 547-550.

URL http://dx.doi.org/10.1016/0167-8396(95)00020-7

[7] U. Reif, A refinable space of smooth spline surfaces of arbitrary topological genus, Journal of Approximation Theory 90 (2) (1997) 174-199.

[8] T. Nguyen, J. Peters, Refinable $C^{1}$ spline elements for irregular quad layout, Computer Aided Geometric Design 43 (2016) 123-130.

[9] H. Bohl, U. Reif, Degenerate Bézier patches with continuous curvature, Computer Aided Geometric Design 14 (8) (1997) 749-761. URL http: //dx.doi.org/10.1016/S0167-8396(97)00005-8

[10] U. Reif, TURBS - topologically unrestricted rational B-splines, Constr. Approx 14 (1995) 57-78.

[11] T. Takacs, B. Jüttler, $\mathrm{H}^{2}$ regularity properties of singular parameterizations in isogeometric analysis, Graphical Models 74 (6) (2012) 361-372. URL http: / /dx.doi.org/10.1016/j.gmod.2012.05.006

[12] J. Peters, U. Reif, Subdivision Surfaces, Vol. 3 of Geometry and Computing, Springer-Verlag, New York, 2008.
[13] F. Cirak, M. Ortiz, P. Schröder, Subdivision surfaces: A new paradigm for thin-shell finite-element analysis, Internat. J. Numer. Methods Engrg. 47 (12) (2000) 2039-2072.

[14] F. Cirak, M. Scott, E. K. Antonsson, M. Ortiz, P. Schröder, Integrated modeling, finite-element analysis, and engineering design for thin-shell structures using subdivision, Computer-Aided Design 34 (2) (2002) 137148.

[15] P. J. Barendrecht, Isogeometric analysis with subdivision surfaces (2013).

[16] T. Nguyen, K. Karčiauskas, J. Peters, A comparative study of several classical, discrete differential and isogeometric methods for solving Poisson's equation on the disk, Axioms 3 (2) (2014) 280-299.

[17] G. Farin, Curves and Surfaces for Computer Aided Geometric Design: A Practical Guide, Academic Press, San Diego, 2002.

[18] H. Prautzsch, W. Boehm, M. Paluszny, Bézier and B-spline techniques, Springer Verlag, 2002.

[19] K. Karčiauskas, J. Peters, Improved shape for multi-surface blends, Graphical Models 8 (2015) 87-98.

[20] K. Karčiauskas, J. Peters, Concentric tesselation maps and curvature continuous guided surfaces, Computer Aided Geometric Design 24 (2) (2007) 99-111. 